\title{
Shock Performance of Different Semiactive Damping Strategies
}

\author{
D. F. Ledezma-Ramirez ${ }^{* 1}$, N. Ferguson ${ }^{2}$, M. Brennan ${ }^{2}$ \\ ${ }^{1}$ Universidad autónoma de Nuevo León \\ San Nicolás de los Garza, México \\ Av. Universidad C.P. 66451 \\ *diego.ledezma@gmail.com \\ ${ }^{2}$ University of Southampton \\ Southampton, United Kingdom \\ University Road C.P. SO17 1BJ
}

\begin{abstract}
The problem of shock generated vibration is presented and analyzed. The fundamental background is explained based on the analysis of a single degree-of-freedom model with passive stiffness and damping. The advantages and limitations of such a shock mount are discussed. Afterwards, different semi-active strategies involving variable damping are presented. These strategies have been used for harmonic excitation but it is not clear how they will perform during a shock. This paper analyzes the different variable damping schemes already used for harmonic vibration in order to find any potential advantages or issues for theoretical shock pulses.
\end{abstract}

Keywords: Damping, shock, vibration.

\section{RESUMEN}

En este artículo se presenta y analiza el problema de vibraciones generadas por impacto, basado en la respuesta de impacto teórica de un sistema de un grado de libertad con rigidez y amortiguamiento pasivos. Se discuten las ventajas y limitaciones de esta configuración. Posteriormente se estudian diferentes estrategias de amortiguamiento variable que han sido usadas en vibración harmonica pero no es claro como se comportarán bajo impacto. Este artículo analiza estas estrategias para encontrar cualquier posible ventaja o problema cuando se usan en situaciones de impacto.

\section{Introduction}

Many practical situations are affected by shock excitation which can be highly detrimental for lot of equipment especially for extremely sensitive objects. The main characteristics of shock excitation are typically high displacements and accelerations applied over a very short time, which causes high mechanical stresses and forces transmitted to the equipment. The subsequent problem of shock isolation is complicated because isolators are required to achieve large deformations in order to store the high amount of energy related to the shock but they should also be stiff enough to withstand the static equipment weight. Most isolators used for shock are passive elements, comprising mechanical springs and rubber elements [1]. Some research on active shock mounts exists, although it is not so extensive; thus, there is a need for more research in shock isolation systems, especially using active or adaptive devices, although passive isolation systems are most widely used because of their cost and reliability.

Normally, the analysis of shock isolation systems is carried out considering single degree-of-freedom damped or undamped systems and this topic has been extensively studied [2]. This model is basically a passive system in which the physical constants of the shock isolator, namely damping, stiffness and damping remain constant. Recently, there has been a growing interest in the use of semiactive isolation systems where the physical constants are able to change during certain conditions in order to provide better vibration isolation. Although these systems have been extensively studied for harmonic and random vibration, there is little work on shock isolation. In this paper, different semiactive approaches previously used for harmonic excitation in the form of variable damping strategies are introduced. The main objective is to evaluate the performance of those systems under shock excitation. 
Different variable damping strategies have been used successfully to address the problem of vibration isolation at high frequencies in heavily damped systems under harmonic excitation. However, in the case of systems subjected to shock, the scenario is different. As the excitation is nonperiodic and only lasts for a short time in comparison with the natural period of the system, the dynamic behavior is quite different in comparison with harmonic excitation and the system is on free vibration after the shock. However, it is important to evaluate the performance of semiactive damping systems not only to find any potential advantages for a specific shock application, but also because many of the studies regarding the topic have not considered shock response. It is not clear how a variable damping system designed for harmonic excitation will perform under shock inputs. As a result, it is critical to find if there are any potential issues regarding the use of such a scheme for shock. The main objective of this paper is to assess the shock isolation performance of existing semiactive damping strategies to find not only possible advantages but also to raise any possible issue in comparison with an equivalent passive model.

\section{Elementary theory}

Consider a single degree-of-freedom viscously damped (MKC) system as shown in Figure 1. The system is subjected to a transient base displacement excitation $u(t)$. The equation of motion is given by

$$
m \ddot{x}+c \dot{x}+k x=c \dot{u}+k u
$$

The term transient means a sudden and short excitation that can be a force, a displacement, an acceleration, etc. and for shock its duration is typically short compared to the natural period $T$ of the linear system. Normally, transient excitations are represented by pulses and steps which can take several shapes [3]. For the purposes of this paper, only the base displacement for two different pulses is considered: a rectangular pulse and a versed sine displacement pulse as shown in Figure 2. These pulses, which have been widely used for shock testing [4] and to evaluate the performance of shock isolation systems, are respectively given by

$$
\begin{gathered}
u(t)=\left\{\begin{array}{ll}
X_{\text {max }} & 0 \leq \mathrm{t} \leq \tau \\
0 & \tau \leq \mathrm{t}
\end{array}\right\} \\
u(t)=\left\{\begin{array}{ll}
\frac{X_{\max }}{2}\left(1-\cos \left(\frac{2 \pi t}{\tau}\right)\right) & 0 \leq \mathrm{t} \leq \tau \\
0 & \tau \leq \mathrm{t}
\end{array}\right\}
\end{gathered}
$$

For the system in Figure 1, one can distinguish between two different responses, the maximax response and the maximum residual response; the former being the maximum shock response at any time and the latter the maximum response once the pulse has finished. The maximax response can be either absolute $(x)$ or relative $(x-u)$.

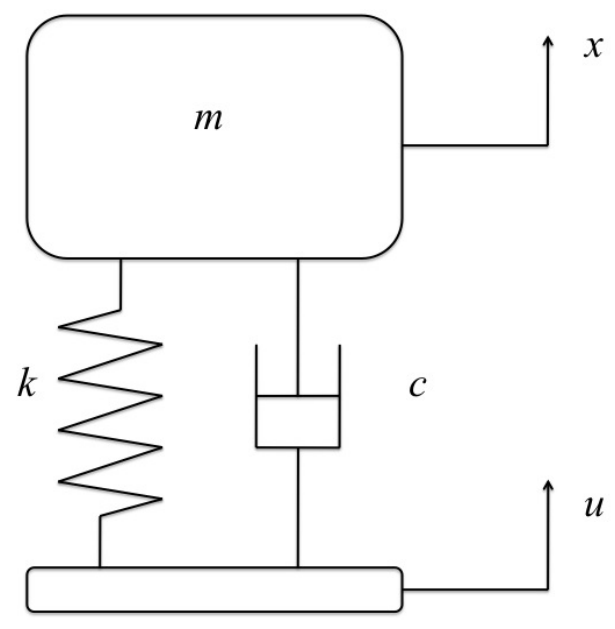

Figure 1. Single degree of freedom system with viscous damping subject to a base displacement transient excitation. 
It is important to note that for some situations, especially for very short pulses, the maximax response occurs after the pulse has finished so both the maximax and maximum residual responses have the same value. In this paper, attention is focused on the maximax response. For very short duration pulses, the shape of the pulse has almost no influence on the response of the system but for long pulses the shape is very important [2]. The response is normalized with respect to the maximum amplitude of the pulse (sometimes this term is referred to as an amplification factor, being analogous to the transmissibility in harmonic excitation), and the pulse duration $\tau$ is normalised with respect to the

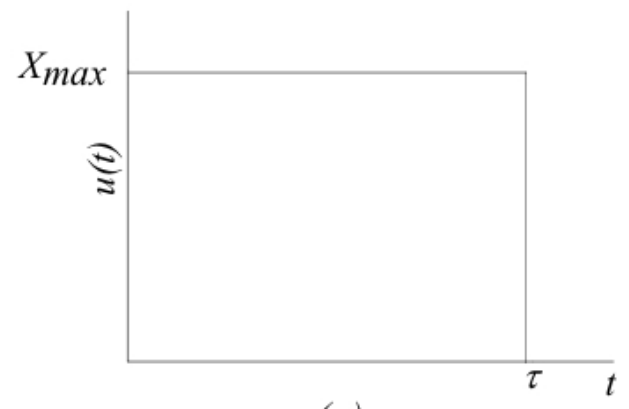

(a) natural period $T$ of the system. Although in this paper only the displacement response is considered for some applications, the acceleration is the parameter of interest but it can be obtained from the absolute and relative displacement [2].

A plot of the normalised displacement response (either maximax or maximum residual) for different values of the normalised pulse duration $(\tau / / T)$ is called the Shock Response Spectrum (SRS) $[1,5]$. This is useful to evaluate the performance characteristics, however, this is not a frequency domain plot, as the name spectrum implies. In fact, it is a time domain plot where every value of the SRS is the maximum response of a corresponding unique system with natural period $T$.

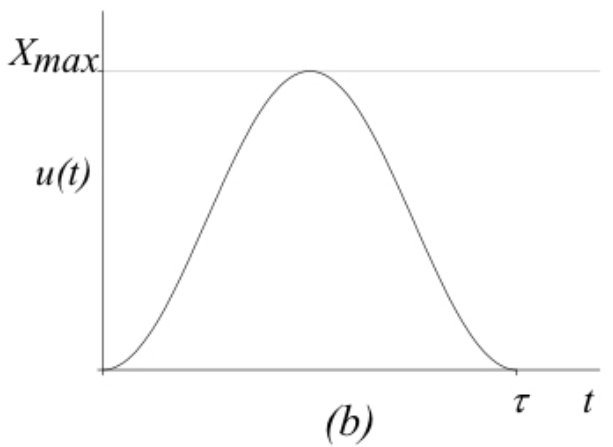

Figure 2. Forcing functions considered: (a) Rectangular pulse, (b) Versed sine pulse.

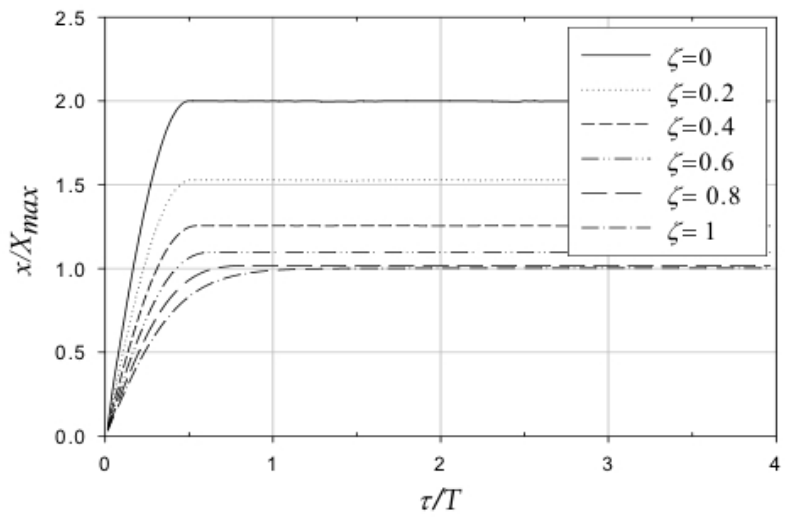

(a)

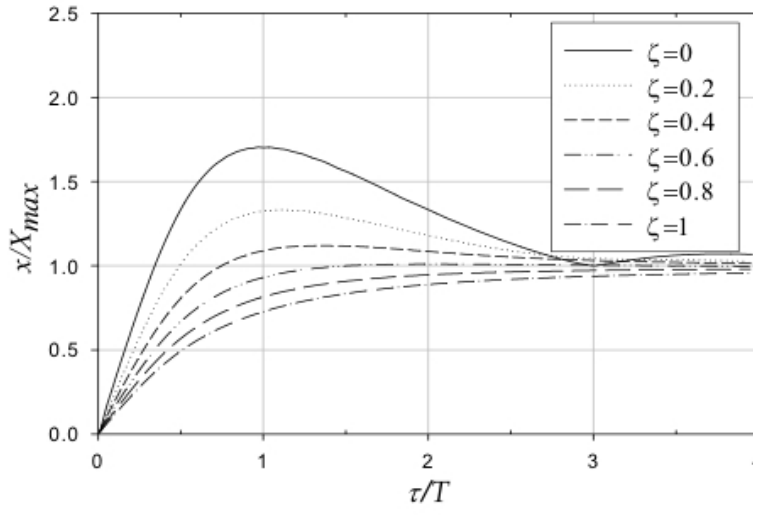

(b)

Figure 3. Shock response spectra of the maximax response for: (a) Rectangular pulse,

(b) Versed sine pulse, for a viscously damped single-degree-of-freedom. 
The SRS of absolute maximax response for the single degree-of-freedom is shown in Figure 3 for both rectangular and versed sine pulses and for various values of the viscous damping ratio $\zeta$. It is clear that the effect of damping in minimizing the response is small; the system has to be highly damped in order to achieve significant reductions in the response. Further insight into the effect of damping can be obtained from Figure 4 which is a plot of the ratio of the maximum value of the shock response spectra for a damped system to the undamped system, for various values of damping.

This plot shows that the effect of damping in limiting the response is small in comparison with the effect of damping in harmonically excited vibrations, for instance. Very high values of damping are required to obtain a considerable reduction in the response. For the undamped system, the maximum for the rectangular pulse is equal to twice the input amplitude, and for the versed sine, it is approximately 1.7 times the input amplitude. These plots have been produced considering the maximum value of the maximax response for each forcing function considered and for other values of $\tau / / T$. Moreover, from the analysis of the SRS presented in Figure 3, it can be seen that effective isolation is only possible when the period ratio $\tau / / T$ is small. This generally requires a very low mounted equipment natural frequency, i.e., a very soft mount for a given equipment mass.

\section{Semiactive control of damping}

In this section, semiactive control of the damping is introduced. Although generally a fully active suspension shows better isolation characteristics, it is more costly and its power consumption can be high [6]. The semiactive mounts are a compromise between a fully active system and a passive system. In this case, if the system fails, one can always have the performance of the passive system.

One of the principal drawbacks of a passive mount is the poor performance at high frequencies in highly damped systems subjected to harmonic excitation. Nonetheless, damping is necessary to minimize the transmissibility at resonance in harmonically excited systems and to reduce the residual vibration after shock inputs. Bearing this in mind, several semiactive control techniques have been developed. One of the most popular is the so-called sky-hook damping [7]. The concept of sky-hook damping is a damper attached between the mass and a fixed reference, as originally proposed by Crosby and Karnopp [7]. The representation is shown in Figure (5a).

This model is useful but it is not always achievable or possible in practice. However, using a controllable damping device, it is possibly to obtain the benefits of the sky-hook concept, as shown in Figure 5(b). The equation of motion for this system is

$$
m \ddot{x}+F_{c}+k x=k u
$$

$F_{c}$ is the variable damping force and its value depends upon the control law used. There are several control laws based on the sky-hook concept (which can be a continuous or on-off skyhook mode). A comparison of some control strategies for harmonic disturbances is given by Liu et al [6].

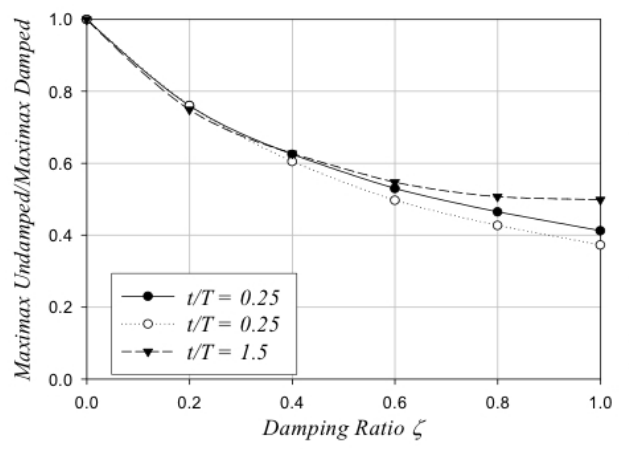

(a)

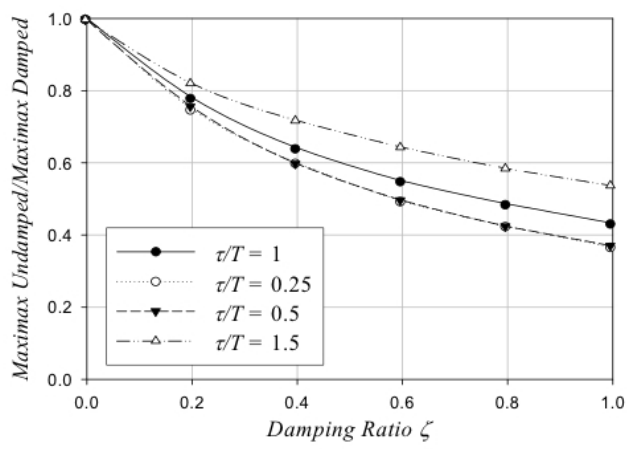

(b)

Figure 4. Effect of damping in the reduction of the displacement response for several values of period ratio $\mathrm{t} / \mathrm{T}$ : (a) Rectangular pulse, (b) Versed sine pulse. 


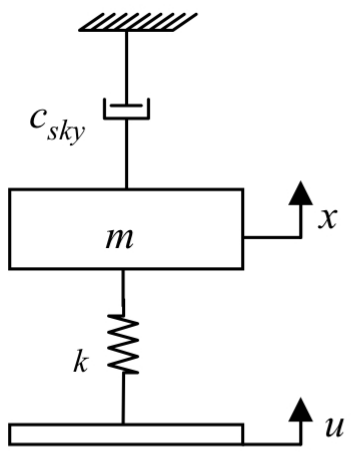

(a)

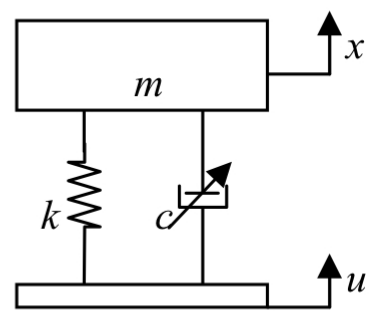

(b)

Figure 5. Theoretical sky-hook concept. (b) Practical implementation of skyhook damping using a variable damping element.

\section{Strategies considered in the analysis}

\subsection{On-off control}

The first control strategy is the one analyzed by Alanoly [6]. The damping force is given by

$$
F_{c}=\left\{\begin{array}{ll}
2 \zeta \omega_{n} \dot{x} & \dot{x}(\dot{x}-\dot{u}) \geq 0 \\
0 & \dot{x}(\dot{x}-\dot{u}) \leq 0
\end{array}\right\}
$$

The damping force depends upon a condition function, which in this case is related to the absolute isolated mass velocity and the relative velocity. If both quantities are in the same direction, the damping force will be applied, otherwise it will be switched off. Equation 7 is used in the numerical simulations, but it is important to note that in practice the damping constant has a nonzero value of minimum damping. This is because there is always a small amount of damping when the damper is in its off state.

\subsection{Balance control}

Another control law is called balance control. This control algorithm is also known as relative control since the variables involved are the relative velocity and the relative displacement [6, 9]. Alanoly and Sankar have stated that the damping force increases the acceleration of the mass during two quarters of a oscillation period whilst in the remaining part of the cycle, the damping force reduces the acceleration of the mass. As the damping force is predominant at high frequencies this will cause a detriment in isolation performance. In order to avoid this effect, the balance control attempts to reduce the damping force in the parts of the cycle where it would cause the acceleration to increase $[6,9]$.

There are two different balance control strategies:

a.On-off balance control .The required damping force is given by

$$
F_{c}=\left\{\begin{array}{ll}
2 \zeta_{\max } \omega_{n} \dot{x} & (x-u)(\dot{x}-\dot{u}) \leq 0 \\
2 \zeta_{\min } \omega_{n} \dot{x} & (x-u)(\dot{x}-\dot{u}) \geq 0
\end{array}\right\}
$$

In this case, the damping force has a nonzero value as in the previous strategy. Instead, the damping force is changed between a maximum and minimum value given by the viscous damping ratio that alternates between a minimum valueand $\zeta_{\min }$ a maximum value $\zeta_{\max }$.

b.Continuous balance control. This strategy is an improvement of the on-off balance control, as given by Alanoly. In the on-off balance control, the damping force is instantaneously equal in magnitude to the spring force, thus, the acceleration is increased. In this improvement, the damping force attempts to cancel the spring force 
[9]. In this case the required restoring force is given by

$$
F_{c}=\left\{\begin{array}{ll}
-k(x-u) & (x-u)(\dot{x}-\dot{u}) \leq 0 \\
0 & (x-u)(\dot{x}-\dot{u}) \geq 0
\end{array}\right\}
$$

i.e. the active 'damper' element acts as a spring with negative stiffness, the damper is adjusted to provide a reaction force equal to the magnitude of the spring force to produce zero acceleration. [6]. Dividing Eq (9) by the relative velocity one can obtain an equivalent viscous damping coefficient given by

$$
c=\left\{\begin{array}{ll}
\frac{-k(x-u)}{(\dot{x}-\dot{u})} & (x-u)(\dot{x}-\dot{u}) \leq 0 \\
0 & (x-u)(\dot{x}-\dot{u}) \geq 0
\end{array}\right\}
$$

i.e. a nonconstant damping coefficient. However, the required viscous damping constant tends to

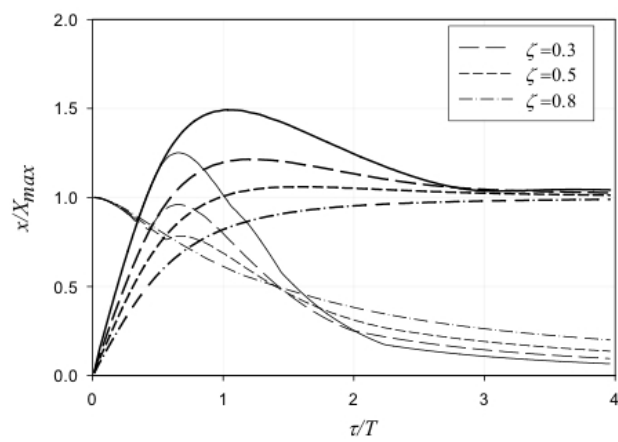

(a)

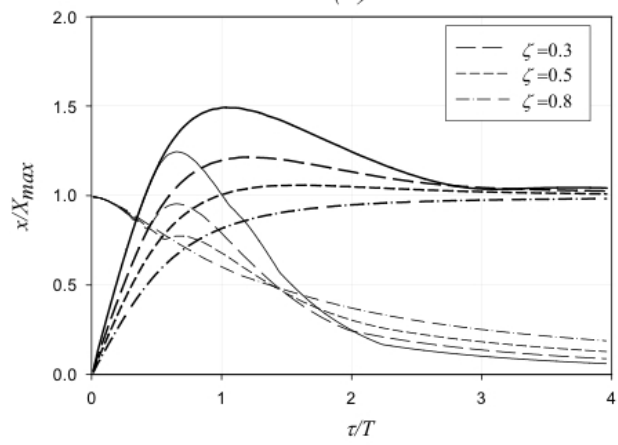

(c) infinity when the relative velocity tends to zero. For a practical implementation and considering nonzero damping, the damping force can be limited and written as

$$
F_{c}=\left\{\begin{array}{ll}
\max \left[c_{\min }, \min \left[\frac{-k(x-u)}{(\dot{x}-\dot{u})}, c_{\max }\right]\right] \dot{x} & (x-u)(\dot{x}-\dot{u}) \leq 0 \\
c_{\min } \dot{x} & (x-u)(\dot{x}-\dot{u}) \geq 0
\end{array}\right\}
$$

The latter condition ensures that there is always a minimum level of damping present. This is valid as all real systems have some amount of damping; moreover, it ensures that the damping force is always between the physical constraints if the relative velocity tends to zero and then the effective damping constant will be infinite. It is important to remember that a real damper is always physically constrained by a minimum and a maximum value. Equation (11) is used for the numerical simulations presented considering a minimum damping so $\zeta=0.1$.

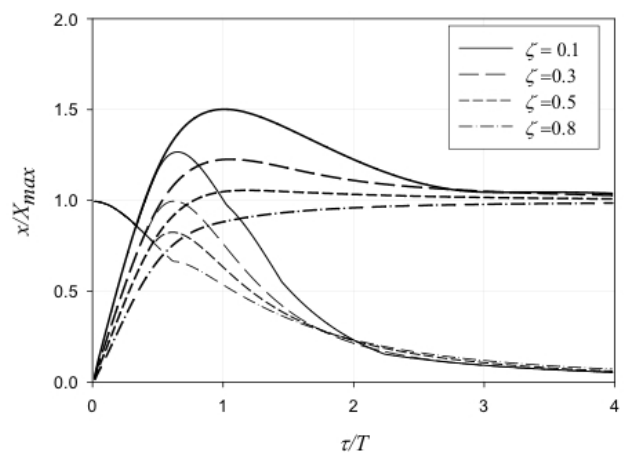

(b)

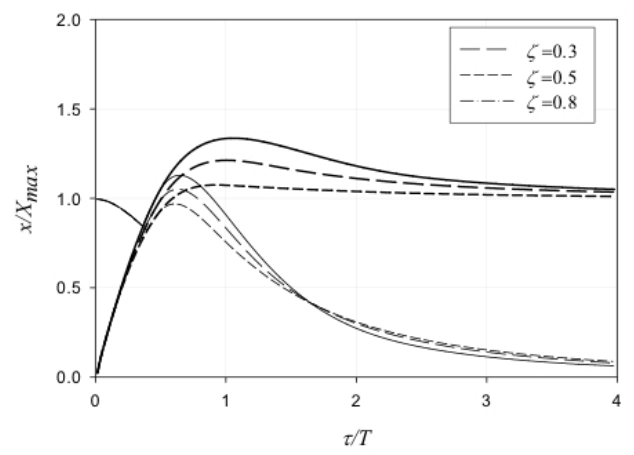

(d)

Figure 6. Shock response spectra for variable damping strategies. (a) Passive system, (b) On off sky hook,

(c) On off balance control,(d) Continuous balance control. Bold lines for absolute maximax response and thin lines for relative maximax response. 


\section{Shock response of the semiactive system}

As mentioned previously, the semiactive strategies considered have been used to solve the compromise of damping and transmissibility in harmonically excited systems; however, in this paper the objective is to evaluate the shock response of these strategies to find any potential advantages or issues. A comparison is made between the three different strategies described. The shock response spectra are used to compare the performance of these approaches and are shown in Figure 6, which comprises the spectra for absolute and relative maximax response. In general, the behavior is very similar for the three strategies. The maximum value of $c$ is chosen so $\zeta=1$. Two-time responses of absolute displacement for the values of $\tau / / T=0.25$ and 1 are presented in Figure 7 . Even though there are small reductions in the relative response compared to the passive systeml they are not very significant and do not justify a more complex semiactive system with variable damping.

Examination of Figure 7 shows that there is an increase in the absolute response for the variable damping strategies considered.

The reason behind this can be explained by examining Figures 8 and 9 which show the variation of the damping ratio and the damping force for each of the strategies considered. While the main interest is to reduce the response during the pulse, the damping remains switched off during most of the pulse duration for all the strategies because the mass velocity and relative velocity have an opposite phase during almost all the pulse duration in the case of the on-off strategy. On the other hand, for balance control the relative quantities (displacement and velocity) both have the same sign for most of the pulse duration and this condition switches off the damper. This would cause the system to behave as a passive undamped system and, even though damping has a little effect in reducing the response, as long as the damper is switched off. the response will increase slightly.

For the residual response, the system undergoes free vibration. For the on-off strategy, as shown in Figures $8(a), 8(b) 9(a)$ and $9(b)$, the result of applying a variable damping force is the same than having a passive damper since it is always switched on (relative velocity and absolute velocity are the same). For the other strategies, there is a small advantage in minimizing residual and relative vibrations. Although one can have a strategy where the damper remains switched on during the pulse, this would be the same than using a passive damper because it makes no sense to switch the damper off after the pulse since it is necessary to minimize residual vibration.

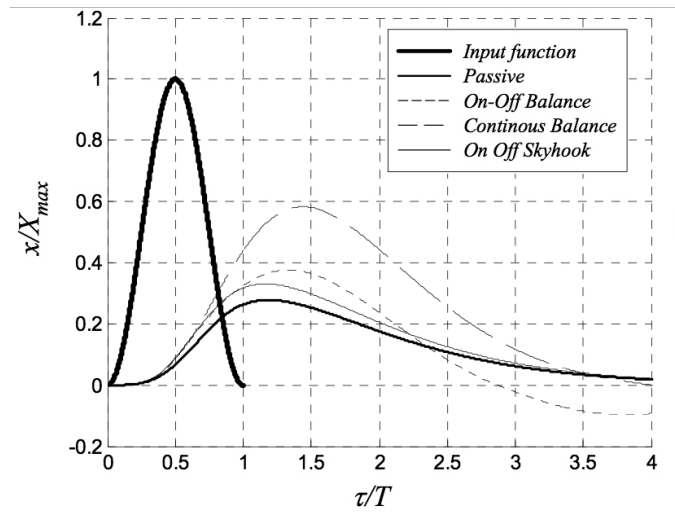

(a)

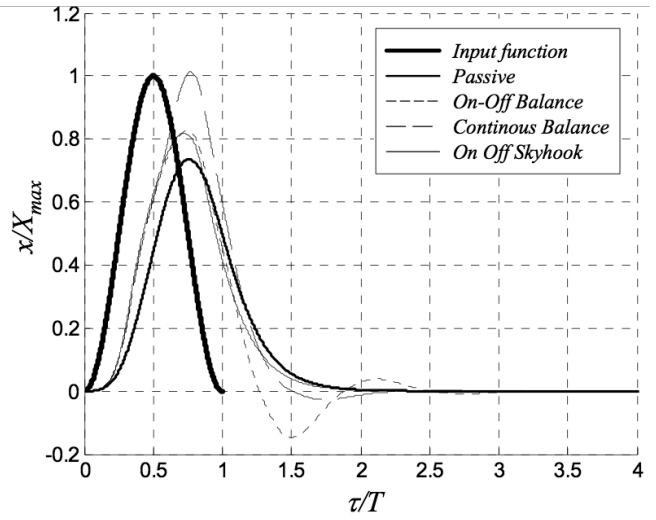

(b)

Figure 7. Time histories for shock response. Comparison between the passive system and the semi active damping strategies for: (a) $\tau / / T=0.25$, (b) $\tau / / T=1$. 


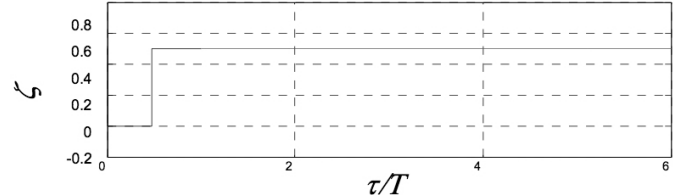

(a)

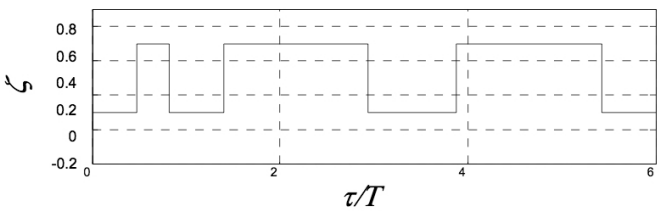

(c)

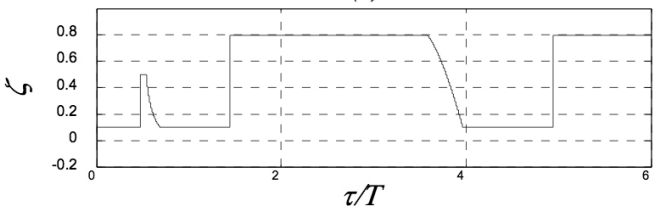

(e)

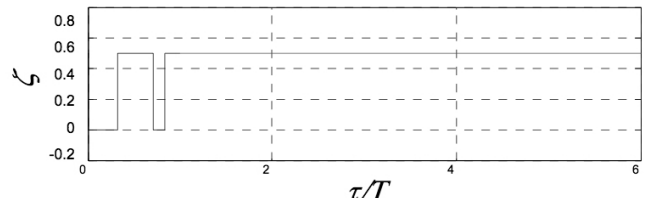

(b)

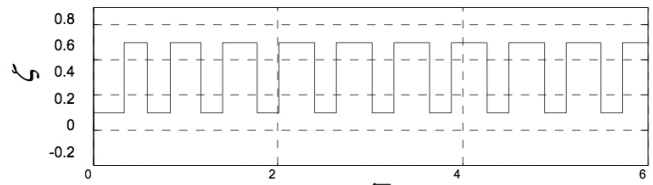

$\tau / T$

(d)

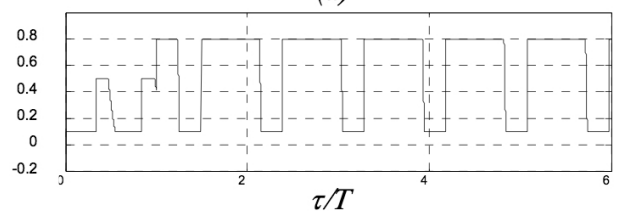

(f)

Figure 8. Variation of the damping ratio for versed sine excitation: (a) On off skyhook control $t / T=0.25$, (b) On off skyhook control $t / \mathrm{T}=1$, (c) on off balance control $t / \mathrm{T}=0.25$, (d) on off balance control $\tau / \mathrm{T}=1$, (e) continuous balance control $T / \mathrm{T}=0.25$, (f) continuous balance control $T / \mathrm{T}=1$.

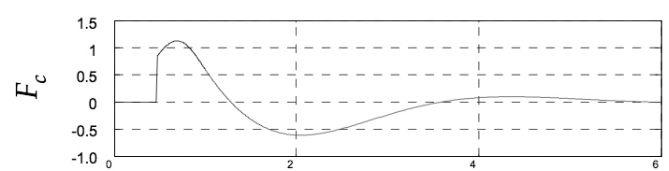

$t / \tau$

(a)

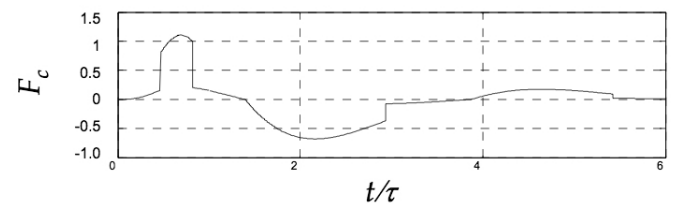

(c)

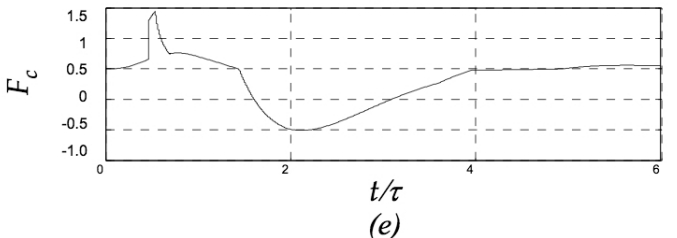

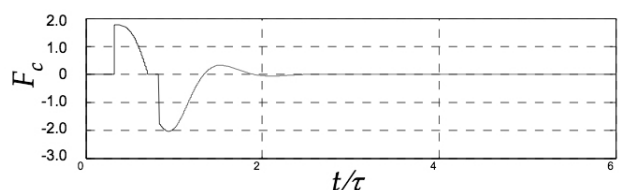

(b)

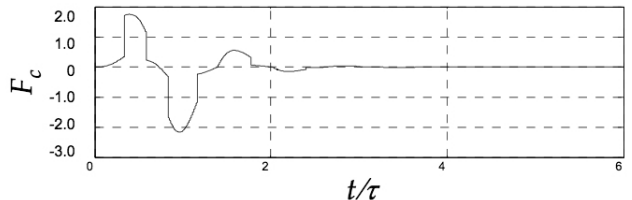

(d)

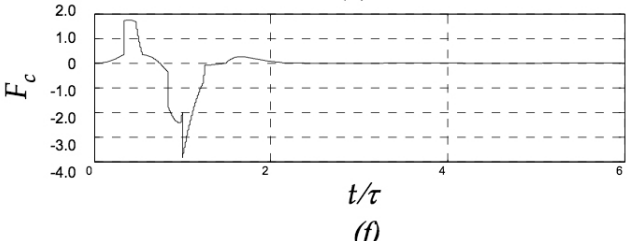

(f)

Figure 9. Variation of the damping force for versed sine excitation: (a) On off skyhook control $t / T=0.25$, (b) On off skyhook control $t / \mathrm{T}=1$, (c) on off balance control $\tau / \mathrm{T}=0.25$, (d) on off balance control $t / \mathrm{T}=1$, (e) continuous balance control $t / T=0.25$, (f) continuous balance control $t / \mathrm{T}=1$. 


\section{Discussion and future work}

The performance of a shock mount is normally assessed using the shock response spectra (SRS), which is a function of the duration of the input rather than the frequency of a periodic excitation. From the fundamental theory presented, it is clear that in order to get shock isolation an isolator of low natural frequency is required, and the damping effect is small in the isolation region of the shock response spectra which occurs when the duration of the input is very small compared to the natural frequency of the system, approximately when the period ratio $\tau / T$ is less than 0.5. As stated in the results presented, there is little change for this situation, as the damping remains turned off during the input for most of the time, either for short or long impacts. For longer pulses, i.e. $\tau / T$ is between 0.5 and 2 damping has some effect in reducing the response and the switching in damping makes almost no difference compared to having the damper on all the time; however, it is important to note that isolation is not worst in any situation under shock pulses compared to the passive case. Still, there could be some issues related to the noncontinuous nature of the damping force as presented in Figure 9 resulting from the sudden swichings in damping. This can potentially lead to sudden acceleration changes or jerks which need to be studied further.

Additionally, another possibility for further improvement of shock isolations using semiactive elements is the use of a variable stiffness element. Since a low stiffness mount is required and damping effect during the shock has a small effect, the possibility of reducing the stiffness during the shock and turning off the damping is a possibility for future work that might produce good results. Moreover, another semiactive strategy could be implemented in order to reduce or minimize quickly the residual vibrations.

\section{Conclusions}

The fundamentals of passive shock excitation and isolation have been reviewed considering the single degree-of-freedom system with viscous damping (MKC). A semiactive approach was used in order to use a variable damping member for shock isolation. Several strategies were considered, namely, on-off sky-hook, continuous and on-off balance controls. The Shock Response Spectra obtained to show that although there are small benefits in using those strategies only for the residual and relative responses, they do not justify the complexity of a semiactive system. This is because the damper remains switched off during almost all the pulse; nevertheless, there are no further issues or disadvantages in the shock response of such strategies and the performance is never worst compared to the passive case, although some sudden acceleration changes may occur.

\section{References}

[1 ] Snowdon, J.C., Vibration And Shock In Damped Mechanical Systems, Wiley and Sons, New York, 1968.

[2 ] Harris, C. M., and Crede, C.E., Shock And Vibration Handbook, McGraw-Hill, New York, 1996.

[3 ] Ayre, R.S., Engineering Vibrations, McGrawHill, New York, 1958.

[4 ] MIL-STD-810D, Environmental Test Methods and Engineering Guidelines, 1983.

[5 ] Mindlin R.D., Dynamics of package cushioning, Bell System Technical Journal, 1945, 24, 353-467.

[6 ] Liu, Y., Waters, T.P., and Brennan, M.J., A comparison of semi active damping control strategies for vibration isolation of harmonic disturbances. Journal of Sound and Vibration, 2005, 280 21-39.

[7 ] Crosby, M.J., and Karnopp, D.C., The active damper: a new concept for shock and vibration control, Shock and Vibration Bulletin, 1973, 43 119-133.

[8 ] Alanoly, J., and Sankar S., Semi-active force generators for shock isolation. Journal of Sound and Vibration, 1988, 126 145-156.

[9 ] Alanoly, J., and Sankar S., A new concept in semi-active vibration isolation, Journal of Mechanisms, Transmissions, and Automation in Design, 1987, 109 242-287. 


\section{Authors' Biographies}

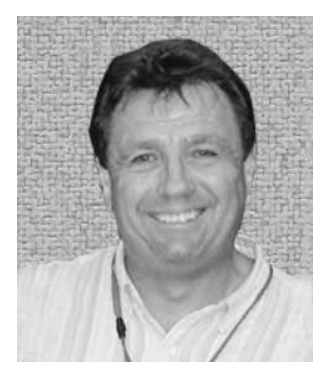

\section{Michael J. BRENNAN}

Michael Brennan holds a personal chair in engineering dynamics and is Chairman of the Dynamics Research Group. He has been at the ISVR since 1995 after leaving the Royal Navy where he enjoyed a career, first as an engineer and then as an academic, spanning a total of 23 years. He graduated from the Open University with first class honors in 1987, received his MSc degree in sound and vibration studies in 1991 and his PhD degree in 1995, both from the Institute of Sound and Vibration Research at the University of Southampton.

Michael Brennan has a wide range of interests in acoustics and vibration including the vibrations of rotating machinery, nonlinear vibrations, vibroacoustics, active and passive control of sound and vibration, energy harvesting and biomimetics. Additionally, he has been a consultant for several companies in the UK and overseas, advising on vibration and acoustic testing methods, control measures and design. Michael Brennan currently teaches on the BEng undergraduate course in Acoustical Engineering, the MSc course in sound and vibration studies. He has also designed, cowritten and copresented several short courses for industry in the UK and abroad. He is a past president of the European Association of Structural Dynamics, associate editor of the Transactions of the ASME Journal of Vibration and Acoustics, guest professor at Harbin Engineering University in China and visiting professor at UNESP in Brazil. He has published over 100 papers in peerreviewed journals and has presented over 140 papers in international conferences.

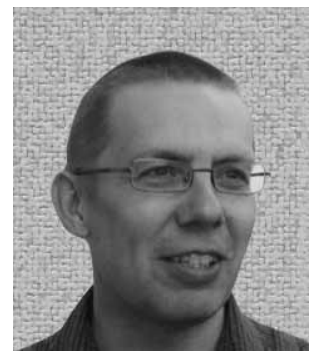

\section{Neil S. FERGUSON}

Neil graduated as a Mathematician in 1980 and then joined ISVR to study for a PhD under Bob White. Apart from a brief spell at University College Swansea, (1984-1985) he has never really been away. Primarily a mathematician/theoretician, his structural dynamics research is very wide ranging including studying trains, planes and brains to name just a few. In between his research activities he has spent the previous ten years (19972007 ) as the undergraduate engineering program organizer and as an academic at ISVR, also juggling his time to organize the Recent Advances in Structural Dynamics conference on three occasions. Now, having relinquished his administration role, he has finally found time to join the IOA and obtain CEng status. His present research includes activities in uncertainty in structural dynamics, vibration control for shock, nonlinear dynamics and applications of wave motion for structural response predictions.

Neil is also a member of the Higher Education Academy, a member of two ESDU (Engineering Sciences Data Unit) committees (Dynamics and Fatigue), have been on the EPSRC Peer Review College, also consultant to British Aerospace (Military Aircraft Division and participated in teaching at other 


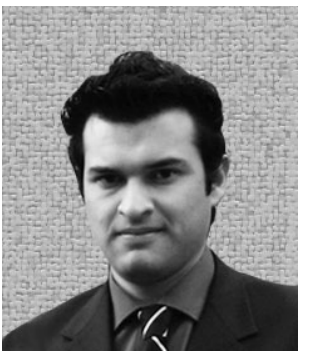

\section{Diego LEDEZMA RAMIREZ}

Diego Ledezma studied electrical and mechanical engineering at the Mechanical and Electrical Engineering School of the Universidad Autónoma de Nuevo León (FIME-UANL) where he graduated presenting a thesis about the relationship between finish surface and mechanical vibration in machine tools. From 2004 to 2008, he underwent postgraduate studies at the Institute of Sound and Vibration of the University of Southampton, obtaining a Ph.D. in 2009. His research project was the study of semiactive and adaptive control strategies to reduce shock generated vibration.

Currently, he is a full time researcher at FIME-UANL where he also teaches at undergraduate and postgraduate levels. He also collaborates with the academic board at school of aeronautics. His main research interests are vibration control using semiactive and non linear elements, structural design, fatigue, and modal analysis. 